Cahiers de recherches médiévales

\title{
Remarques sur la réception des Étymologies d'Isidore de Séville au Trecento
}

Jean-Frédéric Chevalier

\section{OpenEdition}

\section{Journals}

Édition électronique

URL : https://journals.openedition.org/crm/10692

DOI : $10.4000 / \mathrm{crm} .10692$

ISSN : 1955-2424

Éditeur

Honoré Champion

Édition imprimée

Date de publication : 10 décembre 2008

Pagination : 7-16

ISSN : 1272-9752

Référence électronique

Jean-Frédéric Chevalier, «Remarques sur la réception des Étymologies d'Isidore de Séville au

Trecento », Cahiers de recherches médiévales [En ligne], 16 | 2008, mis en ligne le 15 décembre 2011,

consulté le 15 décembre 2022. URL : http://journals.openedition.org/crm/10692 ; DOI : https://

doi.org/10.4000/crm.10692 


\title{
酷M
}

\section{Remarques sur la réception des Étymologies d'Isidore de Séville au Trecento}

\begin{abstract}
Dante meets Isidore of Seville, the Venerable Bede and Richard of St Victor in Canto $X$ of the Paradiso, thus proving that author of the Étymologies was still an unquestioned authority at the beginning of the $14^{\text {th }}$ century. But, a few decades later, his name was rarely mentioned by Petrarch, although Petrarch knew the Étymologies very well.Wondering whether this phenomenon was unique to Petrarch only or whether it was a common one at the time, we attempt to define the reception of the Étymologies in Petrarch's correspondence, in Giovanni Boccaccio's Genealogiae deorum gentilium libri and in Coluccio Salutati's De laboribus Herculis.
\end{abstract}

Résumé : Dante rencontre Isidore de Séville, Bède le Vénérable et Richard de Saint-Victor au chant $X d u$ Paradis. Ainsi l'auteur des Étymologies restait une autorité incontestée au début du XIV siècle. Quelques décennies plus tard, son nom est pourtant rarement mentionné par Pétrarque, même si celui-ci connaissait très bien les Étymologies. Cette approche était-elle caractéristique de Pétrarque ou commune à son époque? Nous nous sommes ainsi interrogé sur la réception des Étymologies dans la correspondance de Pétrarque, dans les Genealogiae deorum gentilium libri de Boccace et dans le De laboribus Herculis de Salutati.

Isidore de Séville apparaît au chant X du «Paradis » dans la Commedia de Dante (vers 130-131) avec Bède le Vénérable et Richard de Saint-Victor. Il semble donc que l'auteur des Étymologies reste une autorité incontestée jusqu'au début du Trecento au moins ${ }^{1}$. Mais Dante ne dit pas explicitement pour quelles œuvres Isidore de Séville figure dans la couronne des sages du quatrième ciel. Bède le Vénérable est certes connu pour son érudition, mais aussi pour ses écrits hagiographiques et théologiques et pour ses poèmes sacrés. Richard de Saint-Victor est quant à lui un théologien mystique. Dans son commentaire de la Commedia de Dante, Benvenuto da Imola précise qu'Isidore de Séville apparaît au chant $\mathrm{X}$ du Paradiso pour son œuvre de spiritualité ${ }^{2}$. Paradoxalement, le nom d'Isidore de Séville n'apparaît pas

\footnotetext{
1 Voir J. Ma. Fernández Catón, «Las Etimologías en la tradición manuscrita medieval estudiada por el prof. dr. Anspach », Archivos Leoneses, 19, 1965, n³7-38, p. 121-384.

${ }^{2}$ Voir Benvenuto da Imola, Comentum super Dantis Aldigherij Comoediam, dans I commenti danteschi dei secoli XIV, XV e XVI, a cura di P. Procaccioli, Roma, Lexis Progetti Editoriali, 1999: Hic autor describit tres animas sanctas simul trium doctorum; et primo Isidori, secundo Bedae, tertio Richardi. Isidorus fuit hispanus episcopus hispalensis, bonus homo, multa scripsit. (Nos recherches ont été grandement facilitées par les ressources mises en ligne par le site www.bibliotecaitaliana.it. Plusieurs de nos citations sont empruntées aux textes édités par ce site). Et Benvenuto da Imola explique pourquoi Dante parle de l'ardente spiro d'Isidore de Séville: l'ardente spiro, scilicet, amore diuino, d'Isidoro, qui fecit librum de summo bono inter alios multos. Le De summo bono est plus connu sous le titre Sententiae. Voir Isidorus Hispalensis, Sententiae, éd. P. Cazier, Turnholti (Corpus Christianorum, Series
}

Cahiers de Recherches Médiévales, 16, 2008 
dans les autres œuvres de Dante ${ }^{3}$. De même, quelques décennies plus tard, son nom est rarement mentionné par Pétrarque. Il nous a semblé intéressant d'essayer de comprendre ce paradoxe. Est-ce un choix propre à Pétrarque ou s'agit-il d'une attitude plus générale au $\mathrm{XIV}^{\mathrm{e}}$ siècle en Italie ? Nous limiterons notre étude aux œuvres de trois auteurs en nous demandant quelle est la réception des Étymologies auprès de Pétrarque (dans sa correspondance), de Boccace (dans la Généalogie des dieux païens) et de Coluccio Salutati (dans le De laboribus Herculis), trois œuvres où la transmission des savoirs est particulièrement significative et où le lecteur s'attend à trouver le nom d'Isidore de Séville.

\section{Le silence de Pétrarque: une attitude singulière au début du Trecento.}

Pétrarque reconnaît qu'il a rarement recours aux Étymologies d'Isidore de Séville. Un exemple est particulièrement significatif dans une des Lettres de la Vieillesse (Rerum senilium, II, 1, 56), adressée à Giovanni da Certaldo. S'interrogeant sur les âges de la vie et notamment sur celui où commence la vieillesse, Pétrarque s'appuie sur l'autorité de Cicéron et d'Augustin 4 . Pour Cicéron, la vieillesse commence à 46 ans tandis que pour Augustin elle débute à 60 ans. Pétrarque ajoute qu'Isidore de Séville situe différemment le début de la vieillesse puisqu'il divise la vie en six âges et que la iuuentus s'achève à 50 ans. Pétrarque ne précise pas cependant que la vieillesse, pour Isidore de Séville (Étymologies, XI, 2,

Latina CXI), 1998. Le nom d'Isidore de Séville n'apparaît qu'une seule fois dans le commentaire de l'Inferno rédigé par Guido da Pisa, mais il est cité dix fois dans le commentaire que Benvenuto da Imola compose sur les trois livres de la Commedia. Pour une autre interprétation, voir P. Dronke, Dante and Medieval Latin Traditions, Cambridge, Cambridge University Press, 1986, p. 96, où l'auteur formule l'hypothèse qu'Isidore de Séville, Bède le Vénérable et Richard de Saint-Victor seraient, tous les trois, auteurs d'un commentaire du Cantique des cantiques. En revanche le nom d'Isidore de Séville est très souvent cité dans un autre commentaire consacré à l'œuvre majeure de Dante : celui composé par Pietro Alighieri. Dans les lignes consacrées aux vers 130-131, Isidore de Séville est mentionné comme l'auteur des Étymologies, Bède comme auteur d'homélies sur les Évangiles et Richard de Saint-Victor est appelé « un grand théologien ».

${ }^{3}$ Pour des allusions par Dante aux œuvres d'Isidore de Séville, voir G. Brugnoli, « Isidoro », Enciclopedia Dantesca, vol. III, Roma, Istituto della Enciclopedia Italiana, 1984 (1971), p. 521-522. G. Brugnoli évoque les hypothèses formulées par A. Vallone dans «Isidoro di Siviglia e Purgatorio XXVIII, 139-44», Studi Danteschi, 35, 1958, p. 259-262 et par P. V. Mengaldo dans Dante Alighieri, De vulgari eloquentia, I, Introduzione e testo, a cura di P. V. Mengaldo, Padova, Antenore, 1968, p. XXXVI n. 1. Pour l'influence des Étymologies d'Isidore de Séville notamment sur la littérature en langue vernaculaire et sur les encyclopédistes médiévaux, nous renvoyons à B. Ribémont, «Brunetto Latini, encyclopédiste et traducteur d'Isidore de Séville. L'ordo et l' «idéologie»: introduction à la matière historique dans le Livre dou Tresor ", Lo scaffale della biblioteca scientifica in volgare (secoli XIII-XVI), Atti del Convegno (Matera, 14-15 ottobre 2004) a cura di Rita Librandi e Rosa Piro, Micrologus' library 16, Firenze, Sismel, 2006, p. 63-79.

${ }^{4}$ Voir Pétrarque, Lettres de la Vieillesse, I-III, édition critique d'E. Nota et traduction de F. Castelli, F. Fabre et A. de Rosny, Paris, Les Belles Lettres, «Les Classiques de l'Humanisme », 2002, p. 134-135. 
5-7 $7^{5}$, ne commence véritablement qu'après 70 ans ${ }^{6}$. L'argument en soi n'est pas d'une grande originalité, mais, au détour de la réflexion de Pétrarque, un détail ne peut que surprendre: Isidorus, quo auctore raro utor... Nous savons pourtant que Pétrarque possédait dès sa jeunesse un manuscrit des Étymologies que son père lui avait offert ${ }^{7}$.

La référence à Isidore de Séville dans cette lettre se limite donc à quelques mots. En outre il s'agit de la seule mention du nom d'Isidore de Séville dans l'ensemble des 18 livres composant les Lettres de la Vieillesse de Pétrarque. Ce désintérêt apparent pour celui qui pourtant n'a cessé d'être une référence érudite tout au long du Moyen Âge apparaît même dans la production antérieure de Pétrarque.

Dans les 24 livres des Lettres Familières, nous ne relevons que trois mentions du nom de l'auteur des Étymologies ${ }^{8}$. Dans la première lettre du livre III (§8), Pétrarque, s'interrogeant sur l'emplacement de l'île de Thulé, rapporte l'opinion d'Isidore de Séville (Étymologies, XIV, 6, 4) mais il ne s'agit peut-être que d'une connaissance indirecte: la référence aux Étymologies a été trouvée dans la Topographia hibernica de Giraud de Barri'. Pétrarque n'a pas spontanément eu recours à l'œuvre d'Isidore de Séville pour compléter ses connaissances. Dans la lettre 14 du livre XV $(\S 25)$, il est seulement fait référence au pays d'origine d'Isidore de Séville. La seule référence intéressante se trouve dans la lettre 4 du livre $X(\S 5)$, lettre adressée à son frère Gherardo, le 2 décembre 1349, sur le thème des liens entre théologie et poésie. Pétrarque s'interroge sur l'origine des mots poeta et uates et il cite, parmi ses sources, les noms de Varron, Suétone et Isidore de Séville $^{10}$. Mais la référence à Isidore de Séville ne peut que décevoir qui cherche des

${ }^{5}$ Pour le texte des Étymologies, nous renvoyons à l'édition publiée par W. M. Lindsay, Oxford, Oxford University Press, 1911.

${ }^{6}$ Voir dans Pétrarque, op. cit., la note 67, p. 386, due à U. Dotti.

${ }^{7}$ Il s'agit du manuscrit de la Bibliothèque Nationale de France lat. 7595. Voir G. Billanovich, La Tradizione del testo di Livio e le origini dell'Umanesimo, Padova, Antenore, 1981, p. 63 64 ; Id., "Dalle prime alle ultime letture del Petrarca », dans Il Petrarca ad Arquà, Atti del convegno di studi nel VI centenario di Petrarca, 1370-1374, a cura di G. Billanovich e G. Frasso, Padova, Antenore, 1975, p.22-23 ; Id., Petrarca letterato: lo scrittoio del Petrarca, Roma, Storia e Letteratura, 1947, p. 124, 265. Dans sa biographie de Pétrarque, U. Dotti rappelle que G. Billanovich propose la date de 1325 pour l'acquisition de ce manuscrit par Pétrarque. Voir U. Dotti, Pétrarque, trad. J. Nicolas, Paris, Fayard, 1991, p. 21, 24. Voir également J. Ma . Fernández Catón, art. cit., p. 286.

${ }^{8}$ Voir également Francesco Petrarca, Le Familiari, edizione critica per cura di Vittorio Rossi, volume quarto per cura di Umberto Bosco: libri XX-XXIV e indici, dans Edizione Nazionale delle Opere di Francesco Petrarca, XIII, Firenze, G. C. Sansoni Editore, 1942, p. 335.

9 Voir Pétrarque, Lettres Familières, I-III, traduction d'A. Longpré, introduction et notes d'U. Dotti, Paris, Les Belles Lettres, «Les Classiques de l'Humanisme », p. 244 et 428 (notes 23 et 26).

${ }^{10}$ Sed si cautius agere mens est, locupletissimos fideiussores ac fide dignissimos testes dabo: primus est Marcus Varro, doctissimus Romanorum; proximus Tranquillus, rerum curiosissimus indagator; tertium non adderem nisi quia is, ut reor, familior est tibi. Horum igitur et Ysidorus, breuiter licet et ipso teste Tranquillo, meminit Ethimologiarum libro octauo. La citation est empruntée à Pétrarque, Lettres familières, VIII-XI, trad. A. Longpré, 
traces des Étymologies dans l'œuvre de Pétrarque: Isidore de Séville n'est mentionné (Étymologies, VIII, 7, 1-3) que parce qu'il transmet la définition que Suétone donne dans son De poetis et Varron dans le De lingua Latina (VII, 36). En outre, Pétrarque ne fait que prolonger le débat engagé en 1315 entre Albertino Mussato et Fra Giovannino de Mantoue sur le statut de la poésie. Les arguments et les garants choisis par Pétrarque ne diffèrent pas de ceux avancés par Albertino Mussato. Quand, à la fin du Trecento, Coluccio Salutati évoquera à son tour la définition des mots poeta et uates (De laboribus Herculis, I, 2, 21-22), il ne fera, lui aussi, que reprendre l'explication proposée par Isidore de Séville, il est vrai relayée par Papias et Balbi comme le suggère B. L. Ullman ${ }^{11}$.

Mais si dans le reste de l'œuvre de Pétrarque le nom d'Isidore de Séville n'apparaît pratiquement pas, nous ne pouvons pas pour autant conclure que l'auteur des Étymologies n'exerce aucune influence sur le poète couronné ${ }^{12}$. Cette influence est cependant diffuse, difficile à déceler puisque Pétrarque peut ne pas citer ses sources $^{13}$; en outre certaines explications étymologiques ne sont pas propres à Isidore de Séville si bien qu'il existe un socle commun de références dont il est parfois inutile, à ses yeux, de préciser l'origine. Il n'en reste pas moins que Pétrarque prend ses distances avec la méthode «étymologique» telle qu'elle est pratiquée par Isidore de Séville. Pétrarque choisit de préférence comme auctores les

notices et notes de Ugo Dotti, Paris, Les Belles Lettres, «Les Classiques de l'Humanisme », 2003 , p. 282-283. Voir également les notes 7 à 10 de la page 585. Pour l'histoire du débat sur le statut du poète et de l'image poétique, voir M. Dazzi, Il Mussato preumanista (1261-1329), L'ambiente e l'opera, Vicenza, Neri Pozza, 1964, p.117-123; E. Garin, Il pensiero pedagogico dell'Umanesimo, Firenze, Giuntine-Sansoni, 1958 ; C. C. Greenfield, Humanist and Scholastic Poetics, 1250-1500, Lewisburg (Pa.), Bucknell university press, 1981; G. Ronconi, Le origini delle dispute umanistiche sulla poesia (Mussato e Petrarca), Roma, Bulzoni, 1976, p. 5-59 ; G. Vinay, "Studi sul Mussato", Giornale Storico della Letteratura Italiana, 126, 1949, p. 113-159.

${ }^{11}$ Colucii Salutatis de laboribus Herculis, éd. B. L. Ullman, Zürich, Thesaurus mundi, 1951, p. 16.

${ }^{12} \mathrm{P}$. Renucci insiste sur les «ascendances médiévales de l'humanisme italien » en prenant l'exemple de l'attitude souvent paradoxale de Pétrarque face à l'autorité d'Isidore de Séville ou d'Uguccione de Pise. Voir P. Renucci, L'aventure de l'humanisme européen au Moyen $\hat{A} g e\left(I V^{e}-X I V^{e}\right.$ siècle), Paris, Les Belles Lettres, «Les Classiques de 1'Humanisme», 1953, p. 163-164 et 190-191. P. Renucci précise notamment que Pétrarque raille, dans son De sui ipsius ignorantia, les affirmations d'Isidore de Séville ou de Vincent de Beauvais sur la description de certaines caractéristiques des éléphants, du crocodile ou de la taupe sans pour autant faire preuve d'esprit plus critique dans d'autres circonstances (op. cit., p. 190, note 195). En réalité Pétrarque répond seulement aux critiques de quatre détracteurs vénitiens qui font étalage d'une vaine érudition. Il ne critique ni Isidore de Séville, ni Vincent de Beauvais mais seulement ceux qui apprennent par cœur des savoirs anecdotiques, voire erronés. Voir Pétrarque, Mon ignorance et celle de tant d'autres, trad. J. Bertrand revue par C. Carraud, notes de C. Carraud, Grenoble, Jérôme Millon, 2000, p. 237-238, note 63.

${ }^{13}$ G. Billanovich indique par exemple que les vers de Martial cités par Pétrarque semblent tirés des Étymologies d'Isidore de Séville. Voir G. Billanovich, Petrarca letterato, op. cit., p. 265. 
grands auteurs classiques, tels que Cicéron et Sénèque, mais aussi les Pères de l'Église comme Ambroise, Augustin et Jérôme. De fait aucune de ses œuvres ne se présente comme une encyclopédie dans la tradition des Étymologies. Il est donc logique que le nom d'Isidore de Séville soit peu cité. Mais la raison essentielle est autre : Pétrarque substitue à l'effort encyclopédique d'Isidore de Séville une autre entreprise: dresser le bilan des connaissances historiques, philosophiques, morales... que l'on peut rassembler à la lecture des plus grands noms de l'Antiquité. Point de départ d'un discours de la méthode "humaniste», l'œuvre de Pétrarque prend quelque distance par rapport au latin très tardif (à l'exception de celui des principaux Pères de l'Église) et médiéval. Pétrarque désire remonter aux sources premières, de telle sorte qu'il omet de parler d'Isidore de Séville ou d'autres lexicographes pour ne citer que Cicéron, Tite-Live, Sénèque... Tous, à cette époque, ont lu Isidore de Séville, tous connaissent son œuvre, mais certains prennent soin de ne pas le nommer de façon à montrer qu'ils portent un autre regard sur l'Antiquité. Significativement dans la lettre 18 du livre III des Lettres Familières, consacrée à sa passion des livres, Pétrarque révèle que c'est à Cicéron qu'il doit son affection pour Varron, Térence, Caton et Xénophon. Il précise tout ce qu'il doit à Sénèque, à Augustin, à Lactance... mais au long de son catalogue des sources antiques n'apparaît jamais le nom d'Isidore de Séville alors que nous savons qu'un manuscrit des Étymologies a été un de ses premiers livres. Le silence de Pétrarque est révélateur des intentions du poète couronné: donner l'impression que c'est seulement au début $\mathrm{du} \mathrm{XIV}^{\mathrm{e}}$ siècle qu'on commence à connaître précisément les auteurs antiques. La découverte à cette époque de manuscrits pendant longtemps oubliés permet, il est vrai, de nourrir cet enthousiasme.

L'attitude de Pétrarque n'est cependant pas celle de tous les premiers humanistes italiens. La définition de la tragédie donnée par Isidore de Séville a été presque la seule, avec celle transmise par Boèce dans la Consolation de Philosophie (II, pr. 2, 12), à traverser le Moyen Âge. Benvenuto da Imola, dans l'introduction de son commentaire à la Commedia de Dante, rappelle ainsi l'autorité d'Isidore de Séville: Potest etiam dici quod sit comoedia, nam secundum Isidorum Comoedia incipit a tristibus et terminatur ad laeta. Et ita liber iste incipit a tristi materia, scilicet ab Inferno, et terminatur ad laetam, scilicet ad Paradisum, siue ad diuinam essentiam $^{14}$.

Cette définition, dont la source principale est le livre VIII des Étymologies, était une référence incontournable en 1315, au moment où Albertino Mussato compose non seulement sa tragédie, mais aussi ses commentaires des tragédies de Sénèque ${ }^{15}$. Pourtant quand il publie en 1900 l'Ecerinis et le commentaire de cette

\footnotetext{
${ }^{14}$ Cette définition était déjà rappelée par Dante lui-même (Épître XIII, adressée à Can Grande, $\S 10$ et De uulgari eloquentia, II, IV, 5-7). Benvenuto da Imola considère d'ailleurs que Comoedia, en dépit de la volonté de Dante, est un titre peu approprié. La Comoedia devrait être appelée Satyra: satyra, idest reprehensoria; reprehendit enim mirabiliter et audacter omnia genera uiciorum, nec parcit dignitati, potestati, uel nobilitati alicuius. Ideo conuenientius posset intitulari satyra, quam tragoedia, uel comoedia. Les modèles littéraires cités sont Horace, Juvénal et Perse.

${ }^{15}$ Dès le début du XIV ${ }^{\mathrm{e}}$ siècle, la définition de la tragédie selon Isidore de Séville paraît insuffisante. Voir Albertino Mussato, Senece uita et mores, édité par A.Ch. Megas,
} 
tragédie réalisé par Guizzardo de Bologne et Castellano de Bassano, Luigi Padrin relève principalement l'influence des Deriuationes d'Uguccione de Pise si bien que le nom d'Isidore de Séville n'apparaît presque pas. En revanche, à la même époque, un commentateur des tragédies de Sénèque, Nicolas Triveth (ou Trevet), n’hésite pas à s'appuyer sur l'autorité de l'auteur des Étymologies. Il se sert essentiellement des Étymologies comme d'un dictionnaire géographique et mythologique. On relève par exemple quinze références aux Étymologies dans le commentaire consacré à Hercule Furieux de Sénèque. Et le nom d'Isidore de Séville apparaît explicitement : ut dicit Ysidorus ${ }^{16}$. L'autorité de l'auteur des Étymologies sert, par exemple, à expliquer pourquoi on parle de deux Éthiopies (Sénèque, Hercule Furieux, vers 38 oet Isidore de Séville, Étymologies, XIV, 5, 16), et ce que signifie le nom propre «les Syrtes» (Sénèque, Hercule Furieux, vers 323 et Isidore de Séville, Étymologies, XIII, 18,6). Si l'autorité d'Isidore de Séville est reconnue en matière de géographie, elle l'est également pour expliquer les noms de divinités de la mythologie. Nicolas Triveth fait ainsi appel aux Etymologies pour expliquer le nom des Furies (Sénèque, Hercule Furieux, vers 93 et Isidore de Séville, Étymologies, VIII, 11, 95), le lien entre les Parques et le destin (Sénèque, Hercule Furieux, vers 188 et Isidore de Séville, Étymologies, VIII, 11, 90-93), ou la nature des Mânes (Sénèque, Hercule Furieux, vers 187 et Isidore de Séville, Étymologies, VIII, 11, 100). On ne relève aucune distance critique par rapport aux affirmations contenues dans les Étymologies. Ce texte est, de fait, le principal ouvrage de référence encyclopédique à la disposition de Nicolas Triveth. Le nom de Papias n'est mentionné qu'une seule fois, pour expliquer le nom propre Lapithes (Sénèque, Hercule furieux, vers 779), de même que celui d'Uguccione de Pise (pour expliquer l'expression ianua Taenari dans Sénèque, Hercule Furieux, vers 587).

La réception des Étymologies dans la Généalogie des dieux païens de Boccace

On ne peut donc dire qu'au début du Trecento l'autorité d'Isidore de Séville soit ouvertement contestée ou sous-estimée. On l'utilise parfois faute de pouvoir trouver une autorité plus sûre. Progressivement les premiers humanistes italiens,

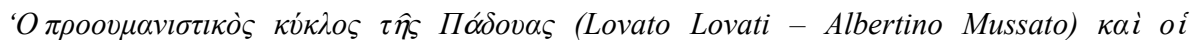

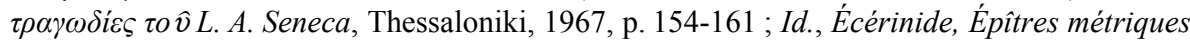
sur la poésie, Songe, éd. J.-F. Chevalier, Paris, les Belles Lettres, "Les Classiques de l'Humanisme », 2000, p. XLIX-LVI ; M. Pastore Stocchi, «Un chapitre d'histoire littéraire aux $\mathrm{XIV}^{\mathrm{e}}$ et $\mathrm{XV}^{\mathrm{e}}$ siècles : Seneca poeta Tragicus ", Les tragédies de Sénèque et le théâtre de la Renaissance, Paris, Éditions du Centre National de la Recherche Scientifique, 1964, p. 11 36. Pour une étude de la transmission de cette définition, voir G. Guastella (con la collaborazione di G. Cardinali), Le rinascite della tragedia, Origini classiche e tradizioni europee, Roma, Carocci, 2006; H. A. Kelly, Ideas and Forms of Tragedy from Aristotle to the Middle Ages, Cambridge, Cambridge University Press, 1993 ; Id., Tragedy and comedy from Dante to pseudo-Dante, Berkeley (Ca.), University of California Press, 1989.

${ }^{16}$ Voir L. Annaei Senecae Hercules furens et Nicolai Treveti expositio, éd. V. Ussani jr, Roma, Edizioni dell'Ateneo, 1959. Les références précises aux Étymologies d'Isidore de Séville sont données par l'éditeur.
} 
notamment Pétrarque et Boccace, chercheront d'autres garants. L'œuvre de Boccace nous apporte un nouvel exemple de réception des Étymologies. Dans l'ensemble des 15 livres de la Généalogie des dieux païens, le nom d'Isidore de Séville apparaît 29 fois $^{17}$. De toute évidence, l'auteur des Étymologies bénéficie d'un meilleur «crédit» auprès de Boccace qu'auprès de Pétrarque. Pourtant Pétrarque et Boccace partagent la même conception de la culture humaniste. Boccace a notamment prolongé la réflexion de Pétrarque sur le statut de l'image poétique et de l'allégorie. Il adopte avec Pétrarque le même esprit critique à l'égard de ses sources. Il est ainsi significatif que le nom d'Isidore de Séville n'apparaisse jamais dans les deux derniers livres de la Généalogie des Dieux païens (livres 14 et 15), consacrés à la défense de la poésie. En revanche dans les autres livres, à l'exception des livres 3 et 6 où il n'apparaît pas, le nom d'Isidore de Séville est mentionné une fois (livres 2, 7, 8,9 ), deux fois (livres 1 et 13), trois fois (livres 5, 10 et 12), mais rarement plus : 5 fois au livre 4 et 7 fois au livre 11 .

Le nom d'Isidore de Séville peut apparaître seul, notamment quand Boccace ne dispose d'aucune autre interprétation pour l'explication d'un nom. Il est ainsi la seule source mentionnée pour l'explication du nom «Tartare» : hunc Tartarum dici uolunt, et hoc secundum Ysidorum ubi de Ethimologiis a tremore frigoris dictum. (Généalogie des Dieux païens, I, ch. XI et Étymologies, XIV, 9, 8), alors que pour évoquer la généalogie du Tartare Boccace fait référence à Theodontius et à Barlaam. En outre Boccace renvoie à Isidore de Séville pour indiquer comment on découvrit Tagès, fils de la Terre (Généalogie des Dieux païens, I, ch. XII et Étymologies, VIII, 9,34 ) ou pour indiquer que la Libye tire son nom de Lybia [sic], fille d'Épaphos et de Cassiopée, et reine de cette partie de l'Afrique (Généalogie des Dieux païens, II, ch. XX et Étymologies, XIV, 5, 1). Isidore de Séville est parfois la seule source nommée pour parler d'un personnage mythologique, comme, par exemple, Aeos, fils de Typhon et fondateur de la cité de Paphos selon certaines traditions (Généalogie des Dieux païens, IV, ch. XXIII et Étymologies, XV, 1, 48). C'est également aux Étymologies que Boccace fait appel pour recenser les noms de tous les vents (Généalogie des Dieux païens, IV, ch. LIV et Étymologies, XIII, 11).

Le nom d'Isidore de Séville apparaît également associé à d'autres noms prestigieux, comme, par exemple, Paul de Pérouse pour l'explication de l'origine des «Muses», filles de Jupiter et de Mémoire. Boccace rappelle qu'Isidore de Séville (Étymologies, III, 15) rattache leur nom à musica : placet Ysidoro, christiano atque san $<c>$ tissimo homini, has Musas appellatas a querendo, eo quod per eas, sicut antiqui uoluerunt, ius carminum et uocis modulatio quereretur, et ob id per deriuationem ab eis musica, que est moderationis peritia, denominata est (Genealogie deorum gentilium, XI, II). Et Boccace de mentionner toutes les autres hypothèses en s'appuyant aussi bien sur l'autorité de Theodontius que sur celle de Macrobe et de Fulgence. Cependant le nom auquel Isidore de Séville est le plus souvent associé est celui de Raban Maur, auteur d'un ouvrage que Boccace intitule De origine (ou originibus) rerum.

\footnotetext{
${ }^{17}$ Voir Giovanni Boccaccio, Genealogie deorum gentilium, dans Tutte le opere di Giovanni Boccaccio, VII-VIII, éd. V. Branca, Milano, A. Mondadori, 1998; Giovanni Boccaccio, Genealogie deorum gentilium libri, éd. V. Romano, Bari, G. Laterza, 1951.
} 
Mais Boccace ne cite pas toujours ses sources. Nous avons déjà indiqué qu'Isidore de Séville n'est pas nommé au livre XIV de la Généalogie des dieux païens. Boccace semble même ignorer que le débat sur le statut du poète inspiré ait commencé avant Pétrarque : le nom d'Albertino Mussato n'apparaît pas. Cependant aux chapitres VII et VIII de ce livre XIV, Boccace revient sur l'origine des mots poesis, poema et uates. Or il est peu vraisemblable qu'il n'ait pas connu la source de cette interprétation étymologique : les Étymologies, VIII, 7, 2. Cet exemple montre que l'autorité d'Isidore de Séville est souvent implicite. Isidore de Séville n'est vraisemblablement pas nommé parce que Boccace enrichit, après Pétrarque, le débat sur l'origine des mots désignant la poésie ou le poète, notamment en faisant de la poésie l'expression d'un feruor. L'autorité suivie n'est plus Isidore de Séville, mais Barlaam, Léonce Pilate et Paul de Pérouse ${ }^{18}$.

Les Étymologies d'Isidore de Séville sont également plus souvent citées que le dictionnaire d'Uguccione de Pise (5 fois), que les Noces de Philologie et de Mercure de Martianus Capella (5 fois), que Papias (12 fois), mais un peu moins souvent mentionnées que le De origine (ou originibus) rerum de Raban Maur. Mais toutes ces sources ne sont que secondaires: en réalité la principale source, mentionnée 241 fois, est Theodontius.

\section{Coluccio Salutati, lecteur des Étymologies?}

Il est intéressant de se demander quel usage, à l'autre extrémité du Trecento, un commentateur comme Coluccio Salutati faisait des Étymologies ${ }^{19}$. Nous avons déjà pu constater que Coluccio Salutati emprunte à Isidore de Séville sa définition des mots définissant le poète. Il tire peut-être également des Étymologies plusieurs définitions touchant à l'art du poète : celle de l'iambe, du pyrrhique et du bacchée (De laboribus Herculis, I, 8, 3-10), mais B. L. Ullman a suggéré que de telles définitions étaient devenues communes, relayées par Papias et Balbi. Le nom d'Isidore de Séville n'est pas mentionné à cet instant par le chancelier de Florence. On ne relève donc que 8 occurrences du nom d'Isidore de Séville, qualifié pourtant de non paruus autor (De laboribus Herculis, II, XVI, 16). La litote est certes un compliment, mais elle valorise peu Isidore de Séville quand on sait que, par exemple, Coluccio Salutati (livre III, ch. XIII) qualifie Claudien de noster uates excultissimus ou Boccace de pater ille meus optimus. Le nom d'Isidore de Séville est seulement mentionné pour rappeler des dissensions entre commentateurs, comme au livre III, ch. XIII où le nom apparaît trois fois : Coluccio Salutati s'interroge sur les raisons pour lesquelles Isidore de Séville (Étymologies, XII, 7, 2720) et Papias sont les seuls à appeler les oiseaux du lac Stymphale du nom <aues $>$ Stromphalides, contrairement à Claudien notamment.

Le nom d'Isidore de Séville est également mentionné pour compléter une interprétation surprenante déjà formulée par d'autres auteurs. Il apparaît ainsi à

\footnotetext{
${ }^{18}$ Voir notamment Giovanni Boccaccio, La Généalogie des Dieux païens, livres XIV et XV: un manifeste pour la poésie, traduit, présenté et annoté par Y. Delègue, Strasbourg, Presses Universitaires de Strasbourg, 2001, p. 42-47 et les notes 51 et 69 p. 87 et 89.

${ }^{19}$ Voir Colucii Salutatis de laboribus Herculis, éd. B. L. Ullman, Zürich, Thesaurus Mundi, 1951.

${ }^{20}$ Mais cette forme n'est attestée que dans un manuscrit, comme l'indique B. L. Ullman.
} 
nouveau associé à ceux de Papias au livre III, ch. XVII ou de Pomponius Mela (livre III, XXIV), ou rapproché de celui de Martianus Capella au livre III, ch. XXIV à propos des Îles des Hespérides. Cet exemple est significatif: Coluccio Salutati rappelle la légende des pommes d'or du Jardin des Hespérides et cite les Étymologies pour justifier l'interprétation rationnelle de ce mythe. La présence d'un dragon, à la forme de serpent, provient de la présence d'un estuaire sinueux dont l'aspect ressemble à celui d'un dragon: Hesperidum insule uocate a ciuitate Hesperide, que fuit in finibus Mauritanie. Sunt autem ultra Gorgodas site sub Athlanteum litus in intimos maris sinus. In quarum ortis fingunt fabule draconem peruigilem aurea mala seruantem. Fertur enim ibi estuarium adeo sinuosis lateribus tortuosum ut uisentibus procul lapsus anguineos imitetur ${ }^{21}$. Comme le rappelle Coluccio Salutati, Isidore de Séville suit une hypothèse déjà formulée par Pline l'Ancien (Histoire naturelle, V, 3), Solin (Recueil de Curiosités, XXIV, 4) et Martianus Capella (Noces de Philologie et de Mercure, VI, 667, 672) ${ }^{22}$. Le nom d'Isidore de Séville apparaît ainsi au sein d'une liste de références. Il n'est jamais référence unique, mais seulement une référence parmi d'autres. Notons également qu'il ne s'agit pas dans cet exemple d'une interprétation fondée sur des hypothèses lexicales, mais d'un effort de «rationalisation » d'un mythe. C'est peut-être la raison pour laquelle ce passage des Étymologies a trouvé grâce aux yeux du chancelier de Florence.

Si le nom d'Isidore de Séville apparaît rarement, B. L. Ullman mentionne en revanche 30 rapprochements possibles entre les commentaires de Coluccio Salutati et les Étymologies; ce qui prouverait que Coluccio Salutati s'inspire naturellement des Étymologies sans rappeler sa source à chaque fois qu'il ne relève aucune controverse sur l'origine d'un mot. En revanche, nous avons vu que le nom d'Isidore de Séville est mentionné quand une autre source avance des hypothèses différentes ou étonnantes sur l'origine d'un mot.

Il semble donc que les Étymologies d'Isidore de Séville demeurent une source encore incontournable au $\mathrm{XIV}^{\mathrm{e}}$ siècle en Italie : les références que l'on trouve dans les textes depuis Boccace jusqu'à Coluccio Salutati en sont une preuve même si on perçoit une prise de distance de plus en plus nette avec cet auctor. On le critique rarement ouvertement, mais on le cite moins, ce qui revient au même. En revanche, les humanistes du $\mathrm{XV}^{\mathrm{e}}$ siècle seront beaucoup plus virulents ${ }^{23}$. Lorenzo Valla critique sévèrement les analyses étymologiques d'Isidore de Séville ainsi que l'ensemble de la lexicographie médiévale ${ }^{24}$. Mais si les humanistes, comme

\footnotetext{
${ }^{21}$ La citation, provenant des Étymologies (XIV, 6, 10), est empruntée à Coluccio Salutati, De laboribus Herculis, III, XXIV, 9, op. cit., p. 301.

${ }^{22}$ Voir notamment Marziano Capella, Le Nozze di Filologia e Mercurio, éd. I. Ramelli, Milano, Bompiani, 2001, p. 929.

${ }^{23}$ Voir F. J. Worstbrock, «Isidor von Sevilla», Die deusche Literatur des Mittelalters. Verfasserlexikon, éd. B. Wachinger et alii, Berlin-New York, W. de Gruyter, t. 11, 2004, col. 717-746. L'auteur mentionne les critiques formulées par Le Pogge et par Lorenzo Valla (art. cit., col. 721).

${ }^{24}$ Voir la Préface au second livre des Elegantiae dans Prosatori latini del Quattrocento, éd. E. Garin, Milano-Napoli, Riccardo Ricciardi Editore, 1952, p. 602-607.
} 
Giovanni Tortelli ou Niccolò Perotti, ont par la suite essayé de bâtir une conception plus scientifique de la lexicographie ${ }^{25}$, il n'en reste pas moins que les Étymologies d'Isidore de Séville fournissent souvent une doxa parfois inavouée.

Jean-Frédéric Chevalier Centre Écritures EA 3943, Université Paul Verlaine - Metz

${ }^{25}$ Voir notamment J.-L. Charlet, «Les instruments de lexicographie latine de l'époque humaniste », Il Latino nell'età dell'Umanesimo, Atti del Convegno, Mantova, 26-27 ottobre 2001, éd. Giorgio Bernardi Perini, Firenze, Leo S. Olschki Editore, 2004, p. 167-195 ; Id., «Tortelli (Giovanni) », Centuriae Latinae II, Cent une figures humanistes de la Renaissance aux Lumière : à la mémoire de Marie-Madeleine de La Garanderie, éd. C. Nativel, Genève, Droz, 2006, p. 807-812. 